\title{
On A New Almost Convergent Sequence Space Defined By The Matrix $\Delta_{u}^{\lambda}$
}

\section{$\Delta_{u}^{\lambda}$ Matrisi Yardımıyla Tanımlanan Yeni Bir Hemen Hemen Yakınsak Dizi Uzayı Üzerine}

\section{Gülsen KILINÇ*}

Adıyaman Üniversitesi, Eğitim Fakültesi, İlköğretim Matematik Öğretmenliği Bölümü,02040, Adıyaman

• Geliș tarihi / Received: 29.10.2019 • Düzeltilerek geliș tarihi / Received in revised form: 03.01.2020 • Kabul tarihi / Accepted: 09.01.2020

\begin{abstract}
In this study, it is defined almost sequence spaces $f(\hat{\Lambda}), f_{0}(\hat{\Lambda})$ and $f s(\hat{\Lambda})$ as domain of the matrix $\Delta_{u}^{\lambda}$. Some topological properties of these spaces are investigated and determined $\beta-, \gamma-$ duals of aforementioned sequence space. Futhermore, it is characterized the class of matrices $(f(\hat{\Lambda}): \mu),(f s(\hat{\Lambda}): \mu),(\mu: f(\hat{\Lambda}))$ and $(\mu: f s(\hat{\Lambda}))$, where $\mu$ is any given sequence space.
\end{abstract}

Keywords: Almost Convergent, Dual Spaces, Matrix Transformations, Matrix Domain of a Sequence Space, Sequence Spaces

$\ddot{\boldsymbol{O}} z$

Bu çalışmada $\Delta_{u}^{\lambda}$ matrisinin etki alanları olarak $f(\hat{\Lambda}), f_{0}(\hat{\Lambda})$ ve $f s(\hat{\Lambda})$ hemen hemen yakınsak dizi uzayları tanımlandı. Bu uzayların bazl topolojik özellikleri incelendi ve $\beta-, \gamma-$ dualleri belirlendi. Ayrica, $(f(\hat{\Lambda}): \mu)$, $(f s(\hat{\Lambda}): \mu),(\mu: f(\hat{\Lambda}))$ ve $(\mu: f s(\hat{\Lambda}))$ matris sinffları karakterize edildi.

Anahtar kelimeler: Hemen Hemen Yakınsaklık, Dual Uzaylar, Matris Dönüşümleri, Bir Dizi Uzayının Matris Etki Alanı, Dizi Uzayları

* Gülsen KILINÇ; gkilinc@adiyaman.edu.tr, Tel: (0530) 870 70 88, orcid.org/0000-0002-9657-2577 


\section{Introduction}

Let us denote space of functionals from $\mathbb{N}$ to $\mathbb{C}$, by $w$, where $\mathbb{N}$ and $\mathbb{C}$ show sets of natural numbers and complex numbers, respectively. When the sequence space is called, it is understood a linear subspace of $w$. The famous classic sequence spaces are $l_{\infty}, c, c_{0}, l_{p}$. These symbols represents sequence space all bounded, convergent, null and absolutely $p$-summable sequences, respectively. Also, we denote the spaces of all bounded and convergent series by $b s$ and $c s$.

Let $A=\left(a_{n k}\right)$ be an infinite matrix of real or complex numbers, $\vartheta$ and $\sigma$ optional sequence spaces. If $x \in \vartheta$ implies that sequence $A x=$ $\left\{(A x)_{n}\right\} \in \sigma$, where sequence $A x$ is the $A$ transform of the sequence $x$ and the general term of this sequence is

$$
(A x)_{n}=\sum_{k} a_{n k} x_{k}
$$

in this case, for each $n \in \mathbb{N}$, the series on the right side of the above equation is convergent. Then we say that the matrix $A$ is a matrix transformation from $\vartheta$ to $\sigma$ and denote it by $A: \vartheta \rightarrow \sigma$. The class of such matrices is showed by $(\vartheta: \sigma)$.

For simplicity in notation, here and in what follows, the summation without limits runs from 0 to $\infty$.

A matrix $E$ is called triangle, if main diagonal's elements aren't zero and elements on the top of the main diagonal are zero. For triangle matrices $E, F$ and a sequence $y$, the equality $E(F y)=$ $(E F) y$ holds. Further, a triangle matrix $W$ uniquely has an inverse $W^{-1}=Z$, also a triangle matrix. The equality $y=W(Z y)=Z(W y)$ yields for talked about matrices.

If there exists a single sequence $\left(t_{k}\right)$ of scalars satisfied the following equation, then the sequence $\left(t_{k}\right)$ is known a Schauder basis (or shortly basis) for a normed sequence space $\vartheta$, where mentioned above equation is, for every $y \in \vartheta$,

$\lim \left\|y-\sum_{n=0}^{k} \alpha_{n} t_{n}\right\|=0$

The series $\sum_{n} \alpha_{n} t_{n}$ which has the sum $y$ is called the enlargement of $y$ according to $\left(t_{k}\right)$ and written as $y=\sum_{n} \alpha_{n} t_{n}$. Schauder basis and algebraic basis coincide for finite sequence spaces.
The matrix domain $\vartheta_{A}$ of an infinite matrix $A$ in a sequence space $\vartheta$ is defined by

$\vartheta_{A}=\left\{y=\left(y_{k}\right) \in w: A y \in \vartheta\right\}$

which is a sequence space. Although in the most cases, the new sequence space is the expansion or the contraction of the original space $\vartheta$, in some cases, these spaces are overlap.

Combined with a linear topology a sequence space $\vartheta$ is denominated a $K$-space, if for each $\vartheta \in \mathbb{N}$, coordinate maps $p_{i}: \vartheta \rightarrow \mathbb{C}$, described by $p_{i}(y)=y_{i}$ are continuous. A $K-$ space which is a complete linear metric space is entitled an $F K-$ space. An $F K$-space whose topology is normable is called a $B K$ - space (Lorentz, 1948) which comprises $\Phi$, the set of all finitely nonzero sequences.

Let us assume that $E$-is a triangle matrix, in that case, we can obviously say that the sequence spaces $\vartheta_{E}$ and $\vartheta$ are linearly isomorphic, i.e., $\vartheta_{E} \cong \vartheta$ and if $\vartheta$ is a $B K-$ space, then $\vartheta_{E}$ is also a $B K$-space with the norm given by $\|y\|_{\vartheta_{E}}=$ $\|E y\|_{\vartheta}$, for all $y \in \vartheta_{E}$. As well as above mentioned sequence spaces $l_{\infty}, c, c_{0}$, and almost convergent sequence space $f$ are $B K$-spaces with the ordinary supnorm described by

$\|y\|_{\infty}=\sup _{k \in \mathbb{N}}\left|y_{k}\right|$

Also $l_{p}$ are $B K-$ spaces with the ordinary norm defined by

$\|y\|_{p}=\left(\sum_{k}\left|y_{k}\right|^{p}\right)^{1 / p},(1 \leq p<\infty)$.

Since the sequence space to be defined is almost convergent sequence space in this study, let's first remember the definition of almost convergent sequence space.

A continuous linear functional $\psi$ on $l_{\infty}$ is said a Banach limit, if

i) For every $y=\left(y_{k}\right), \quad \psi(y) \geq 0$,

ii) $\psi\left(y_{\rho(k)}\right)=\psi\left(y_{k}\right)$, where $\rho$ is shift operatör which is described onto $w$ with $\rho(k)=k+1$, iii) $\psi(e)=1$, where $e=(1,1, \ldots, 1, \ldots)$.

A sequence $y=\left(y_{k}\right) \in l_{\infty}$ is entitled to be almost convergent to generalized limit $l$, if all Banach limits $y$ are $l$ (Lorentz, 1948), and denoted $f-\lim y=l$. In other words, $f-$ limy $=l$ iff uniformly in $n$ 
$\lim _{m \rightarrow \infty} \frac{1}{m+1} \sum_{k=0}^{m} y_{k+n}=l$.

We indicate the sets of all almost convergent sequences by $f$ and series by $f s$ and define as follow:

$f=\left\{y=\left(y_{k}\right) \in w: \lim _{m \rightarrow \infty} s_{m n}(y)=l\right.$, uniformly in $n\}$

where $l$ exists uniformly in $n$,

$s_{m n}(y)=\frac{1}{m+1} \sum_{k=0}^{m} y_{k+n}$,

and

$f s=\left\{y=\left(y_{k}\right) \in w: \exists l \in C \ni\right.$

$\lim _{m \rightarrow \infty} \sum_{k=0}^{m} \sum_{j=0}^{n+k} \frac{y_{j}}{m+1}=$ l uniformly in $\left.n\right\}$.

As known that the containments $c \subset f \subset l_{\infty}$ are precisely acquired. Owing to these containments, norms $\|.\|_{f}$ and $\|.\|_{\infty}$ of the spaces $f$ and $l_{\infty}$ are equivalent. Therefore the sets $f$ and $f_{0}$ are $B K$ spaces having the following norm

$\|y\|_{f}=\sup _{m, n}\left|s_{m n}(y)\right|$

When we look according to summability theory perspective, we can see that to define new Banach spaces by the matrix domain of triangle and investigate their algebraical, geometrical and topological properties is well-known. Therefore, many authors were interested in this subject and by using some known matrices, they did many studies by using some known matrices. Some of them are here:

(Başar et al., 2011; Candan, 2014, 2018; Candan et al., 2015; Karaisa et al., 2015; Kayaduman et al., 2012a,b; Kirisçi, 2012, 2014).

The matrix to be used to construct sequence spaces in this paper is below:

Let $\lambda=\left(\lambda_{k}\right)_{k=0}^{\infty}$ be strictly increasing sequence of positive reals tending to infinity, i.e.

$0<\lambda_{1}<\lambda_{2}<\cdots$ and $\lambda_{k} \rightarrow \infty$, as $k \rightarrow \infty$.

Let $u=\left(u_{k}\right)$ be a sequence such that $u_{k} \neq 0$, for all $k \in \mathbb{N}$. We define the matrix $\hat{\Lambda}=\Delta_{u}^{\lambda}=\left(\hat{\lambda}_{n k}\right)$ as

$\hat{\lambda}_{n k}=\left\{\begin{array}{cl}\frac{\left(\lambda_{k}-\lambda_{k-1}\right)-\left(\lambda_{k+1}-\lambda_{k}\right)}{\lambda_{n}} u_{k}, & \text { if } k<n, \\ \frac{\lambda_{n}-\lambda_{n-1}}{\lambda_{n}} u_{n}, & \text { if } k=n, \\ 0, & \text { if } k>n,\end{array}\right.$
Where

$\hat{\Lambda}_{n}(x)=\frac{1}{\lambda_{n}} \sum_{k=0}^{n}\left(\lambda_{k}-\lambda_{k-1}\right) u_{k}\left(x_{k}-x_{k-1}\right)$

and if $y=\left(y_{k}\right)$ is $\hat{\Lambda}$-transform of a sequence $x=$ $\left(x_{k}\right)$, where for all $k \in \mathbb{N}$

$y_{k}=\sum_{i=0}^{k} \frac{\left(\lambda_{i}-\lambda_{i-1}\right)}{\lambda_{k}} u_{\mathrm{i}}\left(x_{\mathrm{i}}-x_{\mathrm{i}-1}\right)$.

In (Ganie et al., 2013), using the matrix above, the sequence spaces $c_{0}\left(\Delta_{u}^{\lambda}\right)$ and $c\left(\Delta_{u}^{\lambda}\right)$ were defined and investigated. Using the same matrix, we also define the following sequence spaces.

Firstly, let us define sequence spaces $f(\hat{\Lambda})$ and $f_{0}(\hat{\Lambda})$ :

$f(\hat{\Lambda})=\left\{x=\left(x_{k}\right) \in w: y=\left(y_{k}\right)=\hat{\Lambda}(x) \in f\right\}$.

If $y=\left(y_{k}\right) \in \hat{\Lambda}(x) \in f$, it means that $\exists l \in \mathbb{C}$ such that uniformly in $n$,

$$
\begin{aligned}
& \lim _{m \rightarrow \infty} \frac{1}{m+1} \sum_{k=0}^{m} y_{k+n}= \\
& \lim _{m \rightarrow \infty} \frac{1}{m+1} \sum_{k=0}^{m}\left(\sum_{i=0}^{k+n}\left(\frac{\lambda_{i}-\lambda_{i-1}}{\lambda_{k+n}}\right) u_{\mathrm{i}}\left(x_{\mathrm{i}}-x_{\mathrm{i}-1}\right)\right)=l .
\end{aligned}
$$

If $l=0, y=\left(y_{k}\right) \in \hat{\Lambda}(x) \in f_{0}$, and we can define

$f_{0}(\hat{\Lambda})=\left\{x=\left(x_{k}\right) \in w: y=\left(y_{k}\right)=\hat{\Lambda}(x) \in f_{0}\right\}$,

The other sequence space is $f s(\hat{\Lambda})$ :

$f s(\hat{\Lambda})=\left\{x=\left(x_{k}\right) \in w: y=\left(y_{k}\right)=\hat{\Lambda}(x) \in f s\right\}$,

i.e. If $y=\left(y_{k}\right) \in \hat{\Lambda}(x) \in f s$, then $\exists l \in \mathbb{C} \ni$ uniformly in $n$,

$$
\lim _{m \rightarrow \infty} \frac{1}{m+1} \sum_{k=0}^{m} \sum_{j=0}^{k+n} \sum_{i=0}^{j} \frac{\left(\lambda_{i}-\lambda_{i-1}\right)}{\lambda_{j}} u_{\mathrm{i}}\left(x_{\mathrm{i}}-x_{\mathrm{i}-1}\right)=l .
$$

We can redefine the spaces $f s(\hat{\Lambda}), f(\hat{\Lambda})$ and $f_{0}(\hat{\Lambda})$ by the notation of (3), $f s(\hat{\Lambda})=(f s)_{\hat{\Lambda}}$, $f(\hat{\Lambda})=(f)_{\hat{\Lambda}}$ and $f_{0}(\hat{\Lambda})=\left(f_{0}\right)_{\hat{\Lambda}}$.

This paper is organized as following: In chapter 2: some topological properties of defined sequence spaces; in chapter 3: dual spaces of these spaces; 
in chapter 3: the characterization of some matrix classes between these spaces and some classical sequence spaces are given.

\section{Some Topological Properties of These Spaces}

Theorem 2.1:

i) The sequence space $f(\hat{\Lambda})$ is normed space with $\|x\|_{f(\widehat{\Lambda})}$

$$
=\sup _{m, n}\left|\frac{1}{m+1} \sum_{k=0}^{m}\left(\sum_{i=0}^{k+n} \frac{\left(\lambda_{i}-\lambda_{i-1}\right)}{\lambda_{k+n}}\right) u_{i}\left(x_{i}-x_{i-1}\right)\right|
$$

ii) The sequence space $f s(\hat{\Lambda})$ is normed space with with

$$
=\sup _{m, n}\left|\frac{1}{m+1} \sum_{k=0}^{m}\left(\sum_{j=0}^{k+n} \sum_{i=0}^{j} \frac{\|x\|_{f s(\hat{\Lambda})}}{\left.\lambda_{j}-\lambda_{i-1}\right)} u_{i}\left(x_{i}-x_{i-1}\right)\right)\right|
$$

Theorem 2.2: The spaces $f(\hat{\Lambda}), f_{0}(\hat{\Lambda})$ and $f s(\hat{\Lambda})$ are linearly isomorphic to the spaces $f, f_{0}$ and $f s$, respectively, i.e. $f(\hat{\Lambda}) \cong f, f_{0}(\hat{\Lambda}) \cong f_{0}$, and $f s(\hat{\Lambda}) \cong f s$.

Proof: We show that there is a linear transformation between $f(\hat{\Lambda})$ and $f$. Therefore we have to define a transformation from $f(\hat{\Lambda})$ to $f$. Using the matrix $\hat{\Lambda}$, it can be described the transformation $T$ as $T(x)=\hat{\Lambda}(x)$, for each $x \in$ $f(\hat{\Lambda})$. It is easy to see that $T$ is linear. If $T(x)=$ 0 , then $x=0$, so $T$ is one-to-one. Finally, we need to show that $T$ is surjective.

Let us assume $y=\left(y_{k}\right) \in f$ and describe $x=$ $\left(x_{k}\right)$ by

$x_{k}=$
$\sum_{j=0}^{k}\left(\sum_{m=j-1}^{j}(-1)^{j-m} \frac{\lambda_{m}}{u_{j}\left(\lambda_{j}-\lambda_{j-1}\right)} y_{m}\right)$

From here, we have

$$
\begin{aligned}
& \sum_{i=0}^{k}\left(\frac{\lambda_{i}-\lambda_{i-1}}{\lambda_{k}}\right) u_{i}\left(x_{i}-x_{i-1}\right)= \\
& \sum_{i=0}^{k}\left(\frac{\lambda_{i}-\lambda_{i-1}}{\lambda_{k}}\right) u_{i}\left(\sum_{j=0}^{i} \sum_{m=j-1}^{j}(-1)^{j-m} \cdot \frac{\lambda_{m}}{u_{j}\left(\lambda_{j}-\lambda_{j-1}\right)} y_{m}\right)-\sum_{j=0}^{i-1} \sum_{m=j-1}^{j}(-1)^{j-m} \frac{\lambda_{m}}{u_{j}\left(\lambda_{j}-\lambda_{j-1}\right)} y_{m} \\
& =\sum_{i=0}^{k} \frac{\left(\lambda_{i}-\lambda_{i-1}\right)}{\lambda_{k}} u_{i}\left(\sum_{m=i-1}^{i}(-1)^{i-m} \cdot \frac{\lambda_{m}}{u_{i}\left(\lambda_{i}-\lambda_{i-1}\right)} y_{m}\right) \\
& =y_{k}
\end{aligned}
$$

For all $k \in \mathbb{N}$, which leads us to the truth that uniformly in $m$

$f_{\widehat{\Lambda}}-\lim x=f-\lim y$

which implies that $x \in f_{\widehat{\Lambda}}$, consequently, we see that $T$ is surjective. Hence, $T$ is a linear bijection that therefore shows that the spaces $f(\hat{\Lambda})$ and $f$ are linearly isomorphic, as desired. This completes the proof. The fact $f_{0}(\hat{\Lambda}) \cong f_{0}$ can be analogously attested.

Due to the well known fact that the matrix domain $\lambda_{A}$ of the normed sequence space denoted by $\lambda$ has got a base iff the matrix domain $\lambda_{A}$ of the normed sequence space denoted by $\lambda$ has got a base, whenever a matrix $A=\left(a_{n k}\right)$ is a triangle (Jarrah, et al., 1990). (Remark 2.4) and since the space $f$ has no Schauder basis, we have;
Corollary 2.1: The space $f_{\widehat{\Lambda}}$ has no Schauder Basis.

\section{The $\alpha-, \beta-, \gamma-$ Duals Of These Spaces}

The $\alpha-, \beta-, \gamma$-duals of the sequence space $X$ are defined by

$$
\begin{aligned}
& X^{\alpha}=\left\{\begin{array}{c}
a=\left(a_{k}\right) \in w: a x=\left(a_{k} x_{k}\right) \in l_{1}, \\
\forall x=\left(x_{k}\right) \in X
\end{array}\right\} \\
& X^{\beta}=\left\{\begin{array}{c}
a=\left(a_{k}\right) \in w: a x=\left(a_{k} x_{k}\right) \in c s, \\
\forall x=\left(x_{k}\right) \in X
\end{array}\right\} \\
& X^{\gamma}=\left\{\begin{array}{c}
a=\left(a_{k}\right) \in w: a x=\left(a_{k} x_{k}\right) \in b s, \\
\forall x=\left(x_{k}\right) \in X
\end{array}\right\}
\end{aligned}
$$

here $c s$ and $b s$ are defined to be sequence spaces of all convergent and bounded series, respectively. 
Lemma 3.1: (Siddiqi, 1971) So as to the matrix $A$ belongs to the matrix class from $f$ to $l_{\infty}$ is necessary and sufficient condition

$\sup _{n \in \mathbb{N}} \sum_{k}\left|a_{n k}\right|<\infty$

is satisfied.

Lemma 3.2: (S1ddiqi, 1971) So as to the matrix $A$ belongs to the matrix class from $f$ to $c$ is necessary and sufficient conditions:

i) $\quad \sup _{n \in \mathbb{N}} \sum_{k}\left|a_{n k}\right|<\infty$

ii) for each $\mathrm{k} \in \mathbb{N} \quad \lim _{n \rightarrow \infty} a_{n k}=\alpha_{k}$

iii) $\quad \lim _{n \rightarrow \infty} \sum_{k} a_{n k}=\alpha$

iv) $\quad \lim _{n \rightarrow \infty} \sum_{k}\left|\Delta\left(a_{n k}-\alpha_{k}\right)\right|=0$

are satisfied.

Theorem 3.1: The $\gamma$-dual of the space $\mathrm{f}_{\widehat{\Lambda}}$ is the intersection of the sets

$b_{1}=\left\{a=\left(a_{k}\right) \in w: \sup _{n \in N} \sum_{k=0}^{n-1}\left|\hat{a}_{k}(n)\right|<\infty\right\}$,

$b_{2}=\left\{a=\left(a_{k}\right) \in w: \sup _{n \in N}\left|\frac{\lambda_{n}}{u_{n}\left(\lambda_{n}-\lambda_{n-1}\right)} a_{n}\right|<\infty\right\}$.

Proof: For an optional sequence $a=\left(a_{k}\right) \in w$ and take into consideration the following equality.

$\sum_{k=0}^{n} a_{k} x_{k}=$

$\sum_{k=0}^{n}\left\{\sum_{j=0}^{k}\left[\sum_{i=j-1}^{j}(-1)^{j-i} \frac{\lambda_{i}}{u_{j}\left(\lambda_{j}-\lambda_{j-1}\right)} y_{i}\right]\right\} a_{k}=$

$\sum_{k=0}^{n-1} \frac{\lambda_{k}}{u_{k}}\left[\frac{a_{k}}{\lambda_{k}-\lambda_{k-1}}\left(\frac{1}{\lambda_{k}-\lambda_{k-1}}\right.\right.$

$$
\left.\left.-\frac{1}{\lambda_{k+1}-\lambda_{k}}\right) \sum_{j=k+1}^{n-1} a_{j}\right] y_{k}
$$

$+\frac{\lambda_{n}}{u_{n}\left(\lambda_{n}-\lambda_{n-1}\right)} a_{n} y_{n}$

$=\sum_{k=0}^{n-1} \hat{a}_{k}(n) y_{k}+\frac{\lambda_{n}}{u_{n}\left(\lambda_{n}-\lambda_{n-1}\right)} a_{n} y_{n}$
$=D_{n}(y)$

where the general term $d_{n k}$ of the matrix $D$ is determined as follows,

$D=\left(d_{n k}\right)=\left\{\begin{array}{cc}\hat{a}_{k}(n), & k<n, \\ \frac{\lambda_{n}}{u_{n}\left(\lambda_{n}-\lambda_{n-1}\right)} a_{n}, & k=n, \\ 0, & k>n,\end{array}\right.$

for all $k, n \in \mathbb{N}$, where

$$
\begin{aligned}
\hat{a}_{k}(n)=\frac{\lambda_{k}}{u_{k}}\left[\frac{a_{k}}{\lambda_{k}-\lambda_{k-1}}\right. \\
\quad+\left(\frac{1}{\lambda_{k}-\lambda_{k-1}}\right. \\
\left.\left.\quad-\frac{1}{\lambda_{k+1}-\lambda_{k}}\right) \sum_{j=k+1}^{n-1} a_{j}\right] .
\end{aligned}
$$

Thus, we deduce from (4), that $a_{k} x_{k} \in b s$ whenever $x=\left(x_{k}\right) \in f_{\widehat{\Lambda}} \quad$ iff $D y \in l_{\infty}$ whenever $y=\left(y_{k}\right) \in f$, where $D=\left(d_{n k}\right)$ is described in (35). That's why with assistance of Lemma 3.1, $f_{\widehat{\Lambda}}^{\gamma}=b_{1} \cap b_{2}$.

Theorem 3.2: The $\beta$-dual of the space $\mathrm{f}_{\widehat{\Lambda}}$ is the intersection of the sets

$b_{3}=\left\{a=\left(a_{k}\right) \in w: \lim _{n \rightarrow \infty} d_{n k}\right.$ exists $\}$,

$b_{4}=\left\{a=\left(a_{k}\right) \in w: \lim _{n \rightarrow \infty} \sum_{k} d_{n k}\right.$ exists $\}$,

$b_{5}=\left\{a=\left(a_{k}\right) \in w: \lim _{n \rightarrow \infty} \sum_{k} \Delta\left(d_{n k}-\alpha_{k}\right)<\infty\right\}$,

where $\alpha_{k}=\lim _{n \rightarrow \infty} d_{n k}$. Then $f_{\widehat{\Lambda}}^{\beta}=\bigcap_{k=1}^{5} b_{k}$.

Proof: Let us take any sequence $a \in w$. By (4), $a x=\left(a_{k} x_{k}\right) \in c s$ whenever $x=\left(x_{k}\right) \in f_{\widehat{\Lambda}}$ iff $D y \in c$ whenever $y=\left(y_{k}\right) \in f$, where $D=$ $\left(d_{n k}\right)$ is designated in (35). We derive the consequence by Lemma 3.2 that $\left\{f_{\widehat{\Lambda}}\right\}^{\beta}=^{n_{k=1}^{5}} b_{k}$.

Theorem 3.3: The $\gamma$-dual of the space $f s_{\widehat{\Lambda}}$ is the intersection of the sets

$b_{6}=\left\{a=\left(a_{k}\right) \in w: \sup ^{n} \sum_{k} \Delta\left(d_{n k}\right)<\infty\right\}$ 
$b_{7}=\left\{a=\left(a_{k}\right) \in w: \lim _{k \rightarrow \infty} d_{n k}=0\right\}$,

That is, $\left\{f s_{\widehat{\Lambda}}\right\}^{\gamma}=b_{6} \cap b_{7}$.

Proof: This might be acquired in a similar concept as talk about in the proof of theorem 3.1 with lemma 3.1 instead of Lemma 4.2 (iii). So, we neglect details.

Theorem 3.4: Defined the set

$b_{8}=\left\{a=\left(a_{k}\right) \in w: \lim _{n \rightarrow \infty} \sum_{k}\left|\Delta^{2}\left(d_{n k}\right)\right|<\infty\right\}$,

Then, $\left\{f s_{\widehat{\Lambda}}\right\}^{\beta}=b_{3} \cap b_{6} \cap b_{7} \cap b_{8}$.

Proof: This, might be acquired in a similar concept as talk about in the proof of theorem 3.2 with Lemma 3.2 instead of lemma 4.2 (iv). So, we disregard details.

\section{Characterization of Some Matrix Classes}

For shortness, let us write

$a_{n k}=\sum_{j=0}^{n} a_{j k}$

$a(n, k, m)=\frac{1}{m+1} \sum_{j=0}^{m} a_{n+j, k}$

$\Delta a_{n k}=a_{n k}-a_{n, k+1}$

Theorem 4.1: (Başar, 2012) Let $\mu$ be an $F K$ space, $U$ be a triangle matrix, $P=U^{-1}$ and $\eta$ be optional subset of $w$. Then, we have $A=\left(a_{n k}\right) \in$ $\left(\mu_{U}: \eta\right)$ iff for all $n \in \mathbb{N}$,

$C^{(n)}=\left(c_{m k}^{(n)}\right) \in(\mu, c)$

and

$C=\left(c_{n k}\right) \in(\mu, \eta)$,

where

$c_{m k}^{(n)}=\left\{\begin{array}{cc}\sum_{j=k}^{m} a_{n j} p_{j k}, & 0 \leq k \leq m \\ 0, & k>m,\end{array}\right.$

and for all $k, m, n \in \mathbb{N}$,

$c_{n k}=\sum_{j=k}^{\infty} a_{n j} p_{j k}$.

Lemma 4.1: $A \in(f: f)$ iff

i) $\sup _{n \in \mathbb{N}} \sum_{k}\left|a_{n k}\right|<\infty$

ii) $f-\lim a_{n k}=\alpha_{k}$, exist, for each fixed $k \in \mathbb{N}(51)$ iii) $f-\lim \sum_{k} a_{n k}=\alpha$

iv) uniformly in $n \quad \lim _{m \rightarrow \infty} \sum_{k} \mid \Delta[a(n, k, m)-$ $\left.\alpha_{k}\right] \mid=0$,

are satisfied.

For an infinite matrix $A=\left(a_{n k}\right)$, we shall write for shortness that:

$$
\begin{aligned}
d_{m k}^{n}=\tilde{a}_{n k}(m) & =\frac{\lambda_{k}}{u_{k}}\left[\frac{a_{n k}}{\lambda_{k}-\lambda_{k-1}}\right. \\
& +\left(\frac{1}{\lambda_{k}-\lambda_{k-1}}\right. \\
& \left.\left.-\frac{1}{\lambda_{k+1}-\lambda_{k}}\right) \sum_{j=k+1}^{m} a_{n j}\right]
\end{aligned}
$$

where $k<m$.

$d_{n k}=\tilde{a}_{n k}$

$$
\begin{aligned}
=\frac{\lambda_{k}\left[\frac{a_{n k}}{u_{k}}\right.}{\lambda_{k}-\lambda_{k-1}} & \\
& +\left(\frac{1}{\lambda_{k}-\lambda_{k-1}}\right. \\
& \left.\left.-\frac{1}{\lambda_{k+1}-\lambda_{k}}\right) \sum_{j=k+1}^{\infty} a_{n j}\right]
\end{aligned}
$$

$\hat{a}_{n k}=\sum_{i=0}^{n}\left(\frac{\lambda_{i}-\lambda_{i-1}}{\lambda_{k}}\right) u_{i}\left(a_{i k}-a_{i-1, k}\right)$

Theorem 4.2: Let us assume that the entries of the infinite matrices given by $A=\left(a_{n k}\right)$ and

$H=\left(h_{n k}\right)$ are related by the following relation

$h_{n k}=\tilde{a}_{n k}$

for all $k, n \in \mathbb{N}, \mu$ is an orbitrary sequence space. Then $A \in\left(f_{\widehat{\Lambda}}: \mu\right)$ iff for all $n \in \mathbb{N},\left\{a_{n k}\right\}_{k \in \mathbb{N}} \in$ $\left(f_{\widehat{\Lambda}}\right)^{\beta}$ and $H \in(f: \mu)$.

Proof: Let us take an orbitrary sequence space $\mu$ and it is satisfied the condition (56) and recall that $f_{\widehat{\Lambda}}$ and $f$ are linearly isomorphic. We take $A \in$ $\left(f_{\widehat{\Lambda}}: \mu\right)$ and $y=\left(y_{k}\right) \in f$. 
Thus, $H . \hat{\Lambda}$ does exist and $\left\{a_{n k}\right\}_{k \in \mathbb{N}} \in \cap_{k=1}^{5} b_{k}$ which satisfies that $\left\{h_{n k}\right\}_{k \in \mathbb{N}} \in l_{1}$, for each $n \in$ $\mathbb{N}$. Therefore, $H y$ exists and thus for all $n \in \mathbb{N}$

$\sum_{k} h_{n k} y_{k}=\sum_{k} a_{n k} x_{k}$

We have by (56) that $H y=A x$, which leads us to consequence $H \in(f: \mu)$.

Conversely, let $\left\{a_{n k}\right\}_{k \in \mathbb{N}} \in\left(f_{\widehat{\Lambda}}\right)^{\beta}$, for each $n \in \mathbb{N}$ and $H \in(f: \mu)$ satisfy, and take any $x=\left(x_{k}\right) \in$ $f_{\widehat{\Lambda}}$. Then, $A x$ exists. Thus, we acquire from the following equality for each $n \in \mathbb{N}$,

$\sum_{k=0}^{m} a_{n k} x_{k}=$
$\sum_{k=0}^{m}\left[\sum_{j=0}^{k}\left(\sum_{i=j-1}^{j}(-1)^{j-i} \cdot \frac{\lambda_{i}}{u_{j}\left(\lambda_{j}-\lambda_{j-1}\right)} y_{i} a_{n j}\right)\right]$.

As $m \rightarrow \infty$ that $A x=H y$ and this shows that $A \in$ $\left(f_{\widehat{\Lambda}}: \mu\right)$.

Theorem 4.3: $A \in\left(f_{\widehat{\Lambda}}: c\right)$ iff $D^{(n)}=\left(d_{m k}^{(n)}\right) \in$ $(f: c)$ and $D=\left(d_{n k}\right) \in(f: c)$.

Theorem 4.4: $A \in\left(f_{\widehat{\Lambda}}: l_{\infty}\right)$ iff $D^{(n)}=\left(d_{m k}^{(n)}\right) \in$ $(f: c)$ and $D=\left(d_{n k}\right) \in\left(f: l_{\infty}\right)$.

If we change the roles for the spaces $f_{\widehat{\Lambda}}$ and $f$ with $\mu$, we have following theorems.

Theorem 4.5: Assume that the entries of the infinite matrices $A=\left(a_{n k}\right)$ and $L=\left(l_{n k}\right)$ are related by the following relation $l_{n k}=\hat{a}_{n k}$ in (56), for all $k, n \in \mathbb{N}$ and $\mu$ be any given sequence space. Then, $A \in\left(\mu: f_{\widehat{\Lambda}}\right)$ iff $L \in(\mu: f)$.

Proof: Let $x=\left(x_{k}\right) \in \mu$ and take into account the following equality

$$
\begin{gathered}
\{\hat{\Lambda}(A x)\}_{n}=\sum_{i=0}^{n} \frac{\left(\lambda_{i}-\lambda_{i-1}\right)}{\lambda_{k}} u_{i}\left[(A x)_{i}-(A x)_{i-1}\right] \\
=\sum_{i=0}^{n} \frac{\left(\lambda_{i}-\lambda_{i-1}\right)}{\lambda_{k}} u_{i} \sum_{j}\left(a_{i j}-a_{i-1, j}\right) x_{j} \\
=\sum_{j}\left(\sum_{i=0}^{n} \frac{\left(\lambda_{i}-\lambda_{i-1}\right)}{\lambda_{k}} u_{i}\left(a_{i j}-a_{i-1, j}\right)\right) x_{j} \\
=(L x)_{n}
\end{gathered}
$$

which leads us to consequence that $A x \in f_{\widehat{\Lambda}}$ iff $L x \in f$. Thus, proof is completed.
At this time, we are going to denote the following conditions:

for each fixed $k \in \mathbb{N}$

$\lim a_{n k}=\alpha_{k}$, exist

$\lim _{n \rightarrow \infty} \sum_{k} a_{n k}=\alpha$,

$\lim _{n \rightarrow \infty} \sum_{k}\left|\Delta\left(a_{n k}-\alpha_{k}\right)\right|=0$,

$\sup _{n \in \mathbb{N}} \sum_{k}\left|\Delta\left(a_{n k}\right)\right|<\infty$,

for each fixed $n \in \mathbb{N}$,

$\lim _{k \rightarrow \infty} a_{n k}=0$,

$\lim _{n \rightarrow \infty} \sum_{k}\left|\Delta^{2} a_{n k}\right|=\alpha$,

$f-\lim a_{n k}=\alpha_{k}$ exists,

uniformly in $n$

$\lim _{m \rightarrow \infty} \sum_{k}\left|a(n, k, m)-\alpha_{k}\right|=0$,

uniformly in $n$

$f-\lim \sum_{k} a_{n k}=\alpha$,

$\lim _{m \rightarrow \infty} \sum_{k}\left|\Delta\left[a(n, k, m)-\alpha_{k}\right]\right|=0$,

uniformly in $n$

$\lim _{q \rightarrow \infty} \sum_{k} \frac{1}{q+1}\left|\sum_{i=0}^{q} \Delta\left[a(n+i, k)-\alpha_{k}\right]\right|=0$,

$\sup _{n \in \mathbb{N}} \sum_{k}|\Delta a(n, k)|<\infty$,

for each fixed $k \in \mathbb{N}$

$f-\lim a(n, k)=\alpha_{k}$ exists,

uniformly in $n$

$\lim _{q \rightarrow \infty} \sum_{k} \frac{1}{q+1}\left|\sum_{i=0}^{q} \Delta^{2}\left[a(n+i, k)-\alpha_{k}\right]\right|=0$,

$\sup _{n \in \mathbb{N}} \sum_{k}|a(n, k)|<\infty$,

for each fixed $k \in \mathbb{N}$

$\sum_{n} a_{n k}=\alpha_{k}$,

$\sum_{n} \sum_{k} a_{n k}=\alpha$, 
$\lim _{n \rightarrow \infty} \sum_{k}\left|\Delta a(n, k)-\alpha_{k}\right|=0$,

Lemma 4.2: Let $A=\left(a_{n k}\right)$ be an infinite matrix. In that case, the following expressions hold:

i) $\quad A=\left(a_{n}\right) \in\left(l_{\infty}: f\right)$ iff conditions (50), (67) and (68) hold. (Duran, 1972).

ii) $\quad A=\left(a_{n k}\right) \in(f: f)$ iff conditions (50), (67) and (69) hold. (Duran, 1972).

iii) $\quad A=\left(a_{n k}\right) \in\left(f s: l_{\infty}\right)$ iff conditions (64) and (65) hold. (Başar, 2012).

iv) $\quad A=\left(a_{n k}\right) \in(f s: c)$ iff conditions (61), (64) and (66) hold. (Öztürk, 1983).

v) $\quad A=\left(a_{n k}\right) \in(c: f)$ iff conditions (50), (67) and (69) hold. (King, 1966).

vi) $\quad A=\left(a_{n k}\right) \in(b s: f)$ iff conditions (64), (65), (67) and (71) hold. (Başar et al, 1991).

vii) $\quad A=\left(a_{n k}\right) \in(f s: f) \quad$ iff conditions (65), (67) (70) and (71) hold (Başar, 1991).

viii) $\quad A=\left(a_{n k}\right) \in(c s: f)$ iff conditions (64) and (67) hold (Başar et al., 1989).

ix) $\quad A=\left(a_{n k}\right) \in(b s: f s)$ iff conditions (65), (71) and (73) hold (Başar et al., 1991).

x) $\quad A=\left(a_{n k}\right) \in(f s: f s)$ iff conditions (71) and (74) hold (Başar, 1991).

xi) $\quad A=\left(a_{n k}\right) \in(c s: f s)$ iff conditions (72) and (73) hold (Başar et al., 1989).

xii) $\quad A=\left(a_{n k}\right) \in(f: c s)$ iff conditions (75) and (78) hold (Başar, 1989).

Corollary 4.1: The following statements hold:

i) $\quad A=\left(a_{n k}\right) \in\left(f_{\widehat{\Lambda}}: l_{\infty}\right) \quad$ iff $\quad\left\{a_{n k}\right\}_{k \in \mathbb{N}} \in$ $\left(f_{\widehat{\Lambda}}\right)^{\beta}$ for all $n \in \mathbb{N}$ and (50) hold with $\tilde{a}_{n k}$ instead of $a_{n k}$.

ii) $\quad A=\left(a_{n k}\right) \in\left(f_{\widehat{\Lambda}}: c\right) \quad$ iff $\quad\left\{a_{n k}\right\}_{k \in \mathbb{N}} \in$ $\left(f_{\widehat{\Lambda}}\right)^{\beta}$ for all $n \in \mathbb{N}$ and (50), (61), (63) hold with $\tilde{a}_{n k}$ instead of $a_{n k}$.

iii) $\quad A=\left(a_{n k}\right) \in\left(f_{\widehat{\Lambda}}: b s\right) \quad$ iff $\quad\left\{a_{n k}\right\}_{k \in \mathbb{N}} \in$ $\left(f_{\widehat{\Lambda}}\right)^{\beta}$ for all $n \in \mathbb{N}$ and (75) hold with $\tilde{a}_{n k}$ instead of $a_{n k}$. iv) $\quad A=\left(a_{n k}\right) \in\left(f_{\widehat{\Lambda}}: c s\right) \quad$ iff $\quad\left\{a_{n k}\right\}_{k \in \mathbb{N}} \in$ $\left(f_{\widehat{\Lambda}}\right)^{\beta}$ for all $n \in \mathbb{N}$ and (75), (78) hold with $\tilde{a}_{n k}$ instead of $a_{n k}$.

Corollary 4.2: The following statements hold:

i) $\quad A=\left(a_{n k}\right) \in\left(l_{\infty}: f_{\widehat{\Lambda}}\right)$ iff (50), (67) and (68) hold with $\hat{a}_{n k}$ instead of $a_{n k}$.

ii) $\quad A=\left(a_{n k}\right) \in\left(f: f_{\widehat{\Lambda}}\right)$ iff (50), (67), (69) and (70) hold with $\hat{a}_{n k}$ instead of $a_{n k}$.

iii) $\quad A=\left(a_{n k}\right) \in\left(c: f_{\widehat{\Lambda}}\right)$ iff (50), (67) and (69) hold with $\hat{a}_{n k}$ instead of $a_{n k}$.

Corollary 4.3: The following statements hold:

i) $\quad A=\left(a_{n k}\right) \in\left(b s: f_{\widehat{\Lambda}}\right)$ iff (64), (65), (67) and (71) hold with $\hat{a}_{n k}$ instead of $a_{n k}$.

ii) $\quad A=\left(a_{n k}\right) \in\left(f s: f_{\widehat{\Lambda}}\right)$ iff $(65),(67)$ and (71) hold with $\hat{a}_{n k}$ instead of $a_{n k}$.

iii) $\quad A=\left(a_{n k}\right) \in\left(c s: f_{\widehat{\Lambda}}\right)$ iff (64) and (67) hold with $\hat{a}_{n k}$ instead of $a_{n k}$.

Corollary 4.4: The following statements hold:

i) $\quad A=\left(a_{n k}\right) \in\left(b s: f s_{\widehat{\Lambda}}\right)$ iff (65), (71) and (73) hold with $\hat{a}_{n k}$ instead of $a_{n k}$.

ii) $\quad A=\left(a_{n k}\right) \in\left(f s: f s_{\widehat{\Lambda}}\right)$ iff (71) and (74) hold with $\hat{a}_{n k}$ instead of $a_{n k}$.

iii) $\quad A=\left(a_{n k}\right) \in\left(c s: f s_{\widehat{\Lambda}}\right)$ iff (72) and (73) hold with $\hat{a}_{n k}$ instead of $a_{n k}$.

\section{Conclusions}

The purpose of this paper is to define some new almost sequence spaces, to give some properties of these spaces and to determine $\beta-, \gamma-$ duals of these spaces, also to characterize some matrix classes between these spaces and some classical sequence spaces. Studying the domain of generalized difference matrix $\Delta_{u}^{\lambda}$ in the spaces $f, f_{0}, f s$ and determining the $\beta-, \gamma-$ duals of these spaces, characterizing the infinite matrices belongs to the class of matrices $(f(\hat{\Lambda}): \mu),(f s(\hat{\Lambda}): \mu),(\mu: f(\hat{\Lambda}))$ and $(\mu: f s(\hat{\Lambda}))$ - where $\mu$ is any given sequence space-are significant in terms of filling up a gap in the existing literatüre of summability theory. 


\section{References}

Başar, F., 1989. Strongly-conservative Sequence to Series Matrix Transformations. Erciyes Üniversitesi Fen Bilimleri Enstitüsü Dergisi, 5, (12), 888-893.

Başar, F. and Çolak, R., 1989. Almost-conservative Matrix Transformations. Turkish Journal of Mathematics, 13(3), 91-100.

Başar, F., 1991. f-conservative Matrix Sequences. Tamkang Journal of Mathematics, 22(2), 205212.

Başar, F. and Solak, İ., 1991. Almost-coercive Matrix Transformations. Rend. Mat. Appl. 7(11) 249256.

Başar, F. and Kirişçi, M., 2011. Almost Convergence and Generalized Difference Matrix. Computers \& Mathematics with Applications, 61, 602-611.

Başar, F., 2012. Summability Theory and Its Applications, Bentham Science Publishers ebooks. Monographs, xi+405 pp, ISB:978-160805-252-3, İstanbul.

Butkovic, D., Kraljevic, H. and Sarapa, H.N., 1987. On the Almost Convergence, in Functional Analysis, II, Lecture Notes in Mathematics, 1242, 396417, (Springer, Berlin, Germany).

Candan, M., 2012. Domain of the Double Sequential Band Matrix in the Classical Sequence Spaces. Journal of Inequalities and Applications, 2012, 281.

Candan, M., 2014. Some New Sequence Spaces Derived from the Spaces of Bounded, Convergent and Null Sequences, International Journal of Modern Mathematical Sciences, 12(2), 74-87.

Candan, M., 2014. Domain of the Double Sequential Band Matrix in the Spaces of Convergent and Null Sequences, Advances in Difference Equations 2014, 163.

Candan, M., 2014. Almost Convergence and Double Sequential Band Matrix. Acta Mathematica Scientia, 34(2), 354-366.

Candan, M. and Kayaduman, K., 2015. Almost Convergent Sequence Space Reproduced by Generalized Fibonacci Matrix and Fibonacci Core. British Journal of Mathematics \& Computer Science, 7(2), 150-167.

Candan, M., 2018. A New Outlook for Almost Convergent Sequence Spaces. Cumhuriyet Science Journal, 39(1), 34-46.
Duran, J.P., 1972. Infinite Matrices and Almost Convergence. Mathematische Zeitschrift, 128, 75-83.

Ganie, A. and Sheikh, N.A., 2013. On Some New Sequence Spaces of Non-absolute Type and Matrix Transformations. Egyptian Mathematical Society, 21, 108-114.

Jarrah, A.M., and Malkowsky, E., 1990. BK-spaces, Bases and Linear Operators. Rendiconti del Circolo Matematico di Palermo, 2(52), 177-191.

Karaisa, A. and Özger, F., 2015. Almost Difference Sequence Spaces Reproduced by Using a Generalized Weighted Mean. Journal of Computational Analysis and Applications, 19(1), 27-38.

Kayaduman, K. and Şengönül, M., 2012a. On the Riesz Almost Convergent Sequence Space. Abstract and Applied Analysis, 2012, 691694.

Kayaduman, K. and Şengönül, M., 2012b. The Space of Cesaro Almost Convergent Sequence and Core Theorems. Acta Mathematica Scientia, 6, 2265-2278.

King, J.P., 1966. Almost Summable Sequences. Proceedings of the American Mathematical Society, 17, 1219-1225.

Kirisçi, M., 2012. Almost Convergence and Generalized Weighted Mean, AIP Conference Proceedings, 1470, 191-194.

Kirisçi, M., 2014. Almost Convergence and Generalized Weighted Mean II. Journal of Inequalities and Applications, 1, 93.

Lorentz, G. G.,1948. A Contribution to the Theory of Divergent Sequences. Acta Mathematica, 80, 167-190.

Móricz, F. and Rhoades, B.E., 1990. Some Characterizations of Almost Convergence for Single and Double Sequences. Publications de l'Institut Mathématique Nouv S`er, 48(62), 6168.

Öztürk, E.,1983. On Strongly Regular Dual Summability Methods. Communications Faculty of Sciences University of Ankara Series A1 Mathematics Stat. 32, 1-5.

Siddıqi, J.A., 1971. Infinite Matrices Summing Every Almost Periodic Sequences. Pacific Journal of Mathematics, 39(1), 235-251. 